\title{
Design of PC to PC Data Transfer System Using Optical Line of Sight Wireless Communication (OLSWC) Technology
}

\author{
Harish Kalla and M.V. Lakshmaiah ${ }^{2}$ \\ ${ }^{1,2}$ Department of Electronics, Sri Krishnadevaraya University, Anantapur, Andhra Pradesh, India
}

\begin{abstract}
Optical Line of Sight Communication (OLSC) is considered to be future wireless communication technology. OLSC may be used to replace the existing radio frequency communication system and to overcome demerits due to resembling data transmission speed, security and health hazards. OLSC uses high illuminating LEDs as a source of light as well as a communicating source at the same time. To achieve high speed communication in OLSC, using various modulation techniques such as Pulse Width Modulation (PWM), Pulse Position Modulation (PPM) and Orthogonal Frequency Division Multiplexing (OFDM) may be used. In this paper, we propose an optical light communication scheme (OLCS) data transfe using PWM technique for point to point (PC to PC) communication. To evaluate its performance, several tests were conducted based on the parameters such as data transmission (different baud rates), transmitter and receiver distance analysis using different wavelengths of LEDs based on the transmitter and receiver's angle of view. Additionally, we measured the PC to PC communication system performance which resulted the data transmission distance of 3.2 meters at 38400 baud rate and with less bit error rate (BER) $10^{-4}$ without affecting the lighting function.
\end{abstract}

Keywords: Optical Line of Sight Communication, Pulse width modulation, wavelength, point to point communication, data transmission.

\section{Introduction}

Visible light communication (VLC) is one of the fastest improving technology in wireless communication systems. Due to its technological importance scientists and researchers are successfully working on the projects such as European OMEGA project [1], Visible Light Communications Consortium (VLCC) [23] and the Wireless World Research Forum (WWRF) [4] etc. High speed communication [5-6], parallel transmission, sophisticated modulation and demodulation methods [7-8], improving the channel capacity [9-10], etc. are the challenging objectives of this research field. The new trends and improved versions of LED technology enables LED to be twinkling at high speed with more brightness which could be used for illumination as well as communication sources [11]. In future LEDs may be substituted by traditional house and office light sources to overcome the high energy consumption, shorter lifetime and health hazards. VLC with white LEDs have stimulated the wireless communication attention globally.

Universal Serial Bus (USB) Interface is a well-known interface for the computer communications. It matches with almost all devices to be able to connect with a compact data acquisition module, computer and sensors directly. VLC with a variety of real time applications in short- range transmission scenarios is promised to enter into people's daily life. The channel between two computers via VLC has already been achieved in [1213], whereas RS-232 protocol or RS-485 protocol is used to connect visible light module with a PC. Design of an integrated optical receiver using USB interface is introduced by Burton et al. [14]. The data rate and transmission distance can be certainly improved by increasing the number of LEDs and adopting different modulation schemes. An application scenario for the developed system is indoor short distance wireless transmission, where LED with communication function is enveloped as a desk lamp and when turned on, data stream flows wirelessly in both the ways.

In this paper, a point to point data access system is design based on computer USB port and using 1 watt white and red color LEDs. We demonstrate the basic visible light access network using different baud rates. Hyper Terminal and microcontroller programming are used to achieve the transmission of data signals between two PCs with the distance of $60 \mathrm{~cm}$. By considering the background research work on this systems, the present study was developed. Wang Jia-yuan et al. [15], experimentally showed that the data transmission speed achieved was $111.607 \mathrm{kbps}$ for indoor wireless communication distance and can be reached 1.5 meters. In a study conducted by Liwei Ding et al. [16], full duplex VLC system to transmit data, audio and video signals between two computers using USB bridge circuit CH340 and white LED with the speed of $2 \mathrm{Mbps}$, and demonstrated the relationship between system transmission eye diagram and bit rate. Angga Pradana et al. [17], implemented the VLC system using pulse width modulation (PWM) technique to run the text data from PC to LCD. By Measuring the PWM accuracy, distance and angle of view between transmitters to receiver. In a study performed by Angga Pradanaet al[17], line of sight communication system which obtained was $920 \mathrm{bps}$ data transmission speed and $10^{-4}$ bit error rate (BER). Sung-Man Kim et al. [18], developed the half-duplex 
VLCsystem using a led as both a transmitter and a receiver source using different color LEDs. Kaiyun Cui et al.[19],discussed the important issues on practical line of sight (LOS) influence in optical wireless communication and implemented the VLC system using RS232 interface technique, which can operate 3.5 meters horizontal and 1 meter vertical distance by the data rate of $115 \mathrm{Kbps}$.

\section{System Design}

The transmitter PC (Tx-PC) is used to transmit the data stream in ASCII standard. The FT232R driver is used to allow interface the Tx-PC for converting serial UART (TTL level) datato serial data communication. USB signal after conversion has a $3.3 \mathrm{~V}$ peak-to-peak voltage value. These serial data stream is then sent to transmitter microcontroller $(\mathrm{Tx}-\mu \mathrm{C})$ for framing the serial data and fed to transmitter front end circuit (Tx-FEC). The Tx-FEC circuit consist of digital signal modulator (DSM) and LED driver circuit. The digital signal modulator mix the data stream i.e. received the data from microcontroller with carrier signal. The LED driver circuit will toggle the high power LED according to the modulated data and transmits to optical wireless transmission channel. PWMtechnique for Intensity modulation (IM) and line of sight (LOS) scheme is chosen for the widely reception in VLC research.

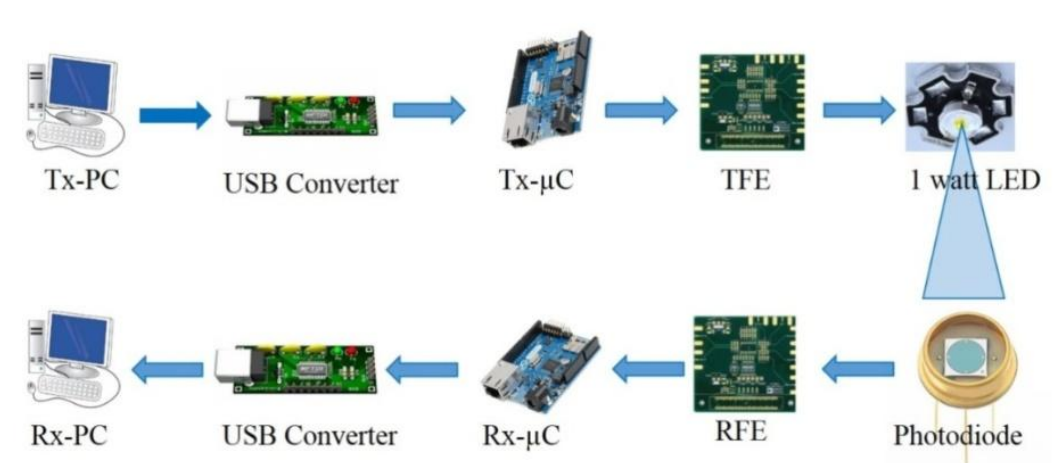

Figure 1.Proposed system transmitter and receiver design

The data will be received by the Si PIN photodiode in the receiver. The information contained in the light is converted into electrical signals. The output signal from the photodiode may affect the noise from attenuation and distortion caused by the channel as well as the influence of photodiode optical nonlinearities characteristic. The received optical signal is sent to receiver front end circuit (Rx-FEC). It contains a custom amplifier LM324 for recovering the electrical signals and DSM to separate the data and carrier signals. A receiver microcontroller $(\mathrm{Rx}-\mu \mathrm{C})$ can decode the data and send to receiver PC through UART to USB converter. The proposed transmitter and receiver system design is presented Figure 1.

\section{Hardware Design}

The transmitter, Tx-FEC comprises digital signal modulator and LED driver, while at the receiver RxFEC consists of an amplifier, signal conditioner and digital signal modulator. The technical specifications of the white LED, LED driver and photodiode used in our experiment are shown in Table I.

Table-I. Technical components specifications of LED, LED driver and Photodiode

\begin{tabular}{|c|l|}
\hline Component & \multicolumn{1}{|c|}{ Specifications } \\
\hline High power LED & Voltage rating: $9-12 \mathrm{~V}$ \\
& Power: $1 \mathrm{~W}$ \\
& $50 \%$ Power Angle: $140^{\circ}$ \\
& Luminous intensity: $80-90 \mathrm{~lm}$ \\
& Wavelength: $400-650 \mathrm{~nm}$ \\
\hline LED Driver IRF 3205 & Fast Switching speed: $50 \mathrm{KHz}$ approximately \\
& Power Dissipation $200 \mathrm{~W}$ \\
& Pulse width $=400 \mu \mathrm{s} ;$ duty cycle $=2 \%$. \\
\hline Photo Diode & Spectral response: $320-110 \mathrm{~nm}$ \\
& Rise time: $1.8 \mu \mathrm{s}$ \\
& Photo sensitivity $(\lambda \mathrm{p}): 0.6 \mathrm{~A} / \mathrm{W}$ \\
\hline
\end{tabular}

\subsection{Transmitter}

At the transmitter side, the LED driver is used to control the LEDs for On-Off switching according to the modulated data signals by a DSM. The schematic diagram of LED driver circuit is shown in Figure 2. This circuit has the advantage that, while being simple, it can be fast switching speed so, quickly turn on and turn off the MOSFET. 


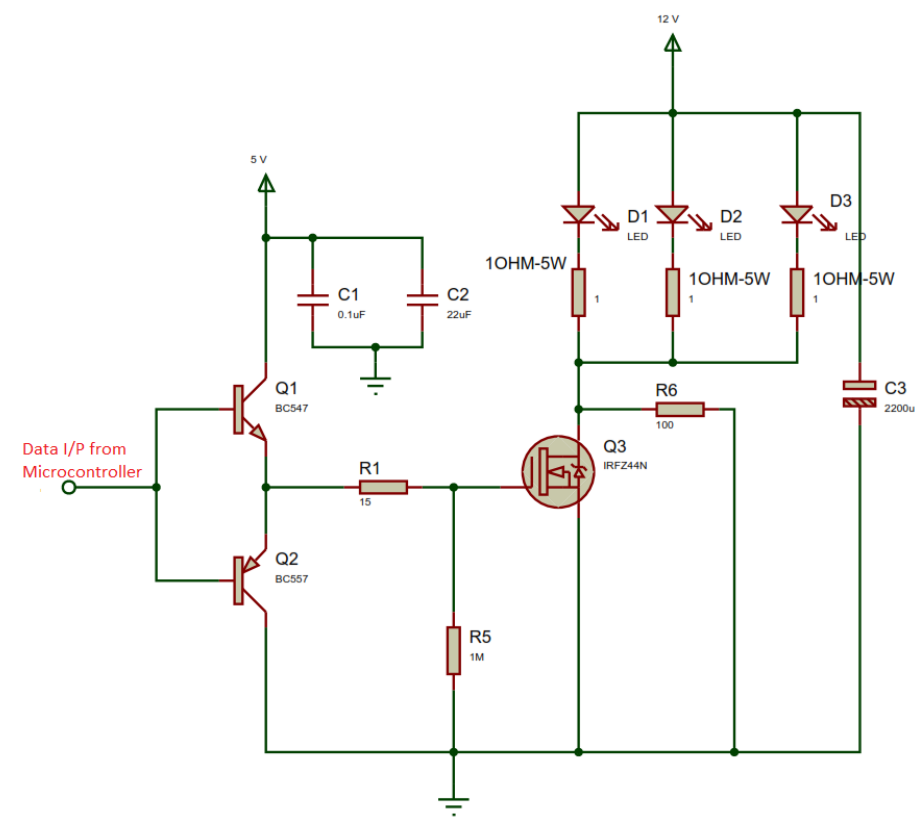

Figure 2. Transmitter front end circuit schematic diagram

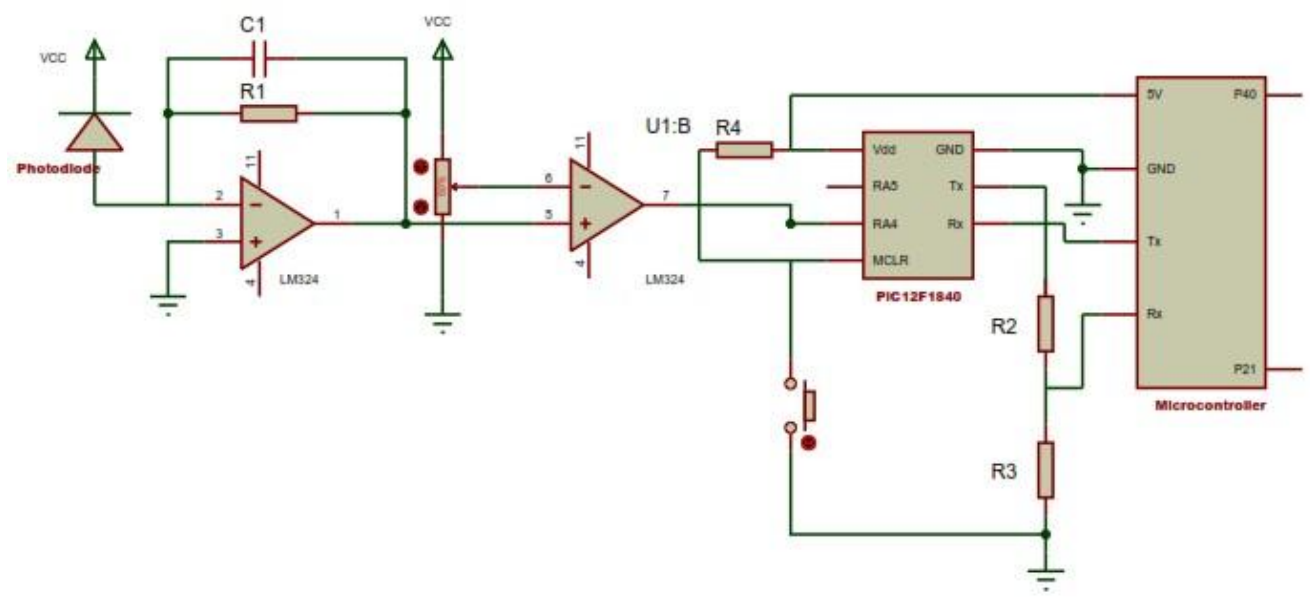

Figure 3. Receiver front end circuit schematic diagram

When the MOSFET is ON, $\mathrm{V}_{\mathrm{GS}}=\mathrm{Vin}-0.7 \mathrm{~V}$ means when DSM output is 5 volts, the MOSFET will be turned on at $4.3 \mathrm{~V}$ only instead of $5 \mathrm{~V}$. This $4.3 \mathrm{~V}$ is enough to fully turn on the MOSFET and when DSM output is zero, MOSFET will be turn OFF. Transistor Q2 and Q3 and is used to amplify the voltage at the output of the DSM, whereas MOSFET Q1 is used to amplify the current.

\subsection{Receiver}

The analog circuit at the receiver side is used to condition the output signal from the photodiode since the output signal of the photodiode will experience attenuation and distortion. For the output of the photodiode is electrical current, Transimpedance amplifier is necessary to convert the current into a voltage. Current to voltage converter is made by the op-amp LM324. The schematic circuit diagram of receiver front end circuit is shown in Figure 3. Once converted into a voltage, this signal is fed to the comparator to overcome the distortion in the signal. Potentiometer can be tuned manually to set the comparator threshold. The output of the comparator is then connected to the DSM to extract the carrier and data signal. Then the demodulated signal is transmitted to $\mathrm{Rx}-\mu \mathrm{C}$ reframing the data to send to the $\mathrm{Rx}-\mathrm{PC}$.

\subsection{Digital signal modulator}

In the DSM, modulated output signals are generated by performing a logical AND operation of both the carrier and modulator signals (from $\mathrm{Tx}-\mu \mathrm{C}$ ) which are provided to the output pin and is called pulse width 
modulation (PWM). The main advantage of this circuit is user friendly. The output ofDSM is to connect any other external circuit for further development and use without changing the DSM program.

\section{Software Design}

All the computers power supply is supported with the 5V DC from USB port. The USB driver software of FT232L firstly installed on PC before being connected to a transmitter and receiver. After transmission baud rate being set and Com port being selected in Hyper Terminal, which is a communication tool available in computer's self-carried enclosures, PC treats the transmitter and receiver modules as a visual serial port via which text data can be transmitted. The program is designed for transmitter and receiver microcontrollers and DSM. The hardware setup is illustrated in Figure 4.

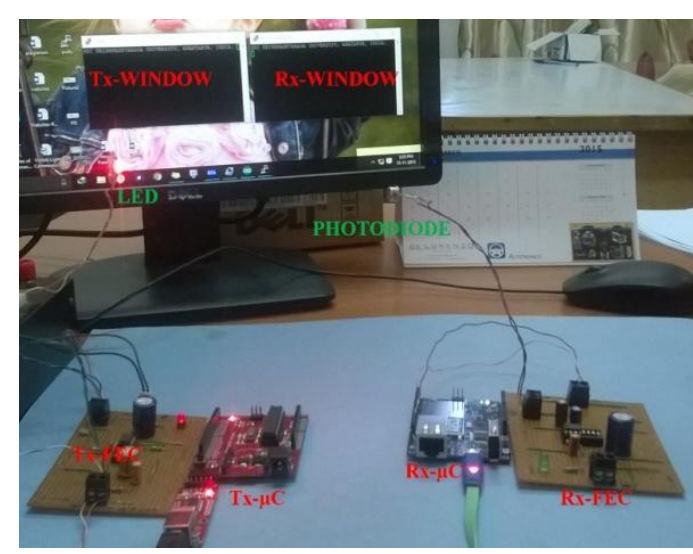

Figure 4. Illustration of practical hardware setup

The Embedded-C program is designed on $\mathrm{Tx}-\mu \mathrm{C}$ consists of data buffer, data conversion from ASCII into the bit stream, data framing for synchronization, and data modulation is done by DSM microcontroller. The modulated data is then connected to the LED driver. The data packet is received when the start bit starts detecting and the data reception process will be terminated when the stop bit is detected. The data packet is then demodulated and converted into ASCII characters which displays on the receiver PC.The transmission between two computers using Hyper Terminal is shown inFigure 5.

\section{Test and Analysis}

Several tests were performed in this experiment. To ensure that the front end circuits works properly, we observed the pulse shape at the Tx-FEC and Rx-FEC. This test is important to examine whether the signals from the transmitter can be received by receiver so that the following process is feasible to be performed. Other testing parameters such as transmitter and receiver distance, the angle of position is varied to determine the effect to the modulation accuracy. Bit error rate (BER) is calculated to measure the performance quality of data streaming test.

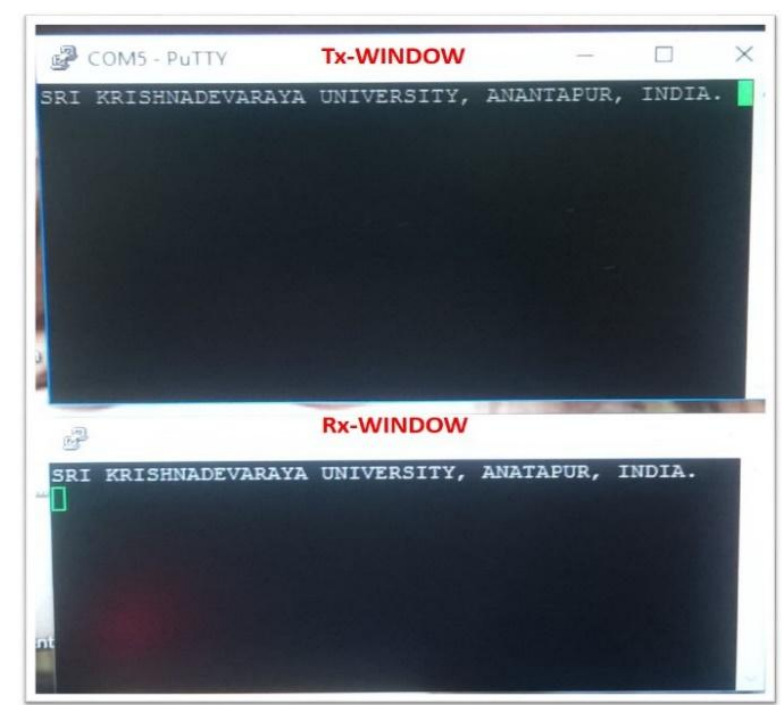

Figure 5.HyperTerminal windows for transmitted and received data 


\subsection{Hardware Test}

The front end circuits are tested by passing data signals at the transmitter and observing the shape of the pulse at the receiver. Figure 6 showstransmitted data signals at the Tx-FEC and the output of the Rx-FEC. The Tx-FEC is modulated the data signals and send to optical free space via LED driver. The Rx-FEC circuit photodiode receives the data signals and will to condition the signal and demodulatesinto an exact dataformat with the compensation on delay effect between the transmitted and received pulses, as shown in Figure 6 . However, the delay time is very small10-50 $\mu$ s, hence, can be accepted or ignored.

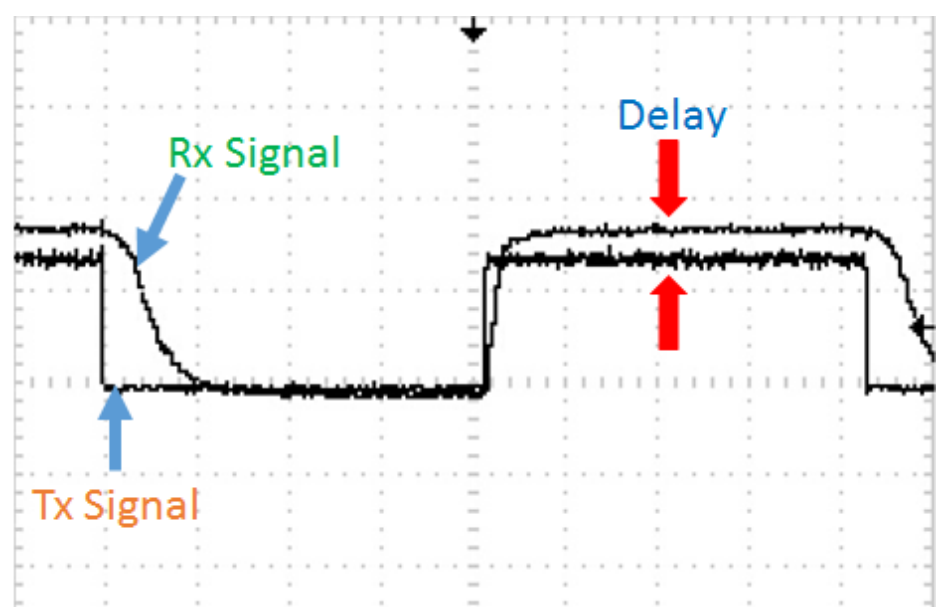

Figure 6.Delay between transmitter and receiver DFE

\subsection{The Effect of Distance}

Obviously, as one kind of wireless communication, VLC must also be able to provide data communication with high mobility to the user. One of the parameters that determines the mobility is the distance variable between the transmitter and receiver. In general, we can conclude that the longer the distance between LED and PD, the modulation accuracy gets worse. This is due to the fact that, longer distance will make PD receive diminishing light intensity. In this experiment we usedone watt red and white colour LEDs to transfer data. The red colour LED, due to its high wavelength $(620-630 \mathrm{~nm})$ and luminous intensity $(20-50 \mathrm{~lm})$ transmits the data signals up to $65 \mathrm{~cm}$ without error at 38400 baud rate.But it was found to be 38400 baud rate at $45 \mathrm{~cm}$ distance without error with white LED.

\subsection{Effect of Receiver's Angle of View}

Another parameter to support high mobility is the varying angle (position) of the light reception. In the previous tests, the position of the LED and PD are in a straight line LOS with zero angle. In the present case, the angle of photodetector reception is changed from $0^{\circ}$ to $60^{\circ}$. From the test results the rising in the reception angle does not always lead to a decrease in accuracy. This is due to the fact that the multipath effect is not only influences by this configuration (compared to LOS configuration), but also, the environmental conditions. However, in general, increasing the angle of view will worsen the modulation accuracy.

\subsection{Baud rate vs. distance performance}

Table-II clearly shows that, the speed of the simplex channel is related to the distance of the transmitter using white LED and the Photodiode at receiver. For a faster baud rate and with no errors we need to decrease the distance (i.e., 38,400 Baudrate) for almost $45 \mathrm{~cm}$. If we increase the distance, we need to reduce the baud rate (i.e. 9600 Baud rate) for almost $60 \mathrm{~cm}$.

Table-II. Transmitter and receiver distance vs. baud rate performance

\begin{tabular}{|c|c|}
\hline Baud rate & Max Distance for tolerablebit errors $(\mathrm{cm})$ \\
\hline 9600 & 60 \\
\hline 19200 & 56 \\
\hline 28800 & 49 \\
\hline 38400 & 45 \\
\hline
\end{tabular}

\section{Conclusions And Feature Work}

We developed a PC to PC data communication using optical wireless system prototype to achieved the transmission between two computers using different wavelengths of light LEDs using PWM technique with different baud rates.The red colour LED, transmitted the data signals up to $65 \mathrm{~cm}$ without error at 38400 baud 
rate.But it was found to be 38400 baud rate at $45 \mathrm{~cm}$ distance without error with white LED, and achieved the very small amount of the delay is $10-50 \mu$ s. To achieve longer distances, the more optimizing circuit designs with optical lenses, efficient coding method, advanced modulation techniques and mechanism for correcting errors will be considered to improvement the transmission system in our future work.

\section{References}

[1]. "Home Gigabit Access project," funded by European Framework 7.'” http://www.ict-omega.eu/.

[2]. VLCC, "Visible Light Communications Consortium," 2008.

[3]. M. Nakagawa, "Visible Light Communications," 2007.

[4]. "Wireless World Research Forum." http://www.wireless-world-research.org/.

[5]. J. Vucic, C. Kottke, S. Nerreter, K. Habel, A. Buttner, K. D. Langer and J. W. Waleski, "125 Mbit/s over 5 m Wireless Distance by Use of OOK-Modulated Phosphorescent White LEDs," Processing of $35^{\text {th }}$ European Conference of Optical Communication, 20-24 September 2009.

[6]. Y. Zheng and M. Zhang, "Visible Light Communications Recent Progresses and Future Outlooks," Processing of Photonics and Optoelectronics Conference, 2011, pp. 1-6.

[7]. C. H. Yeh, Y. F. Liu, C. W. Chow, Y. Liu, P. Y. Huang and H. K. Tsang, "Investigation of 4-ASK Modulation with Digital Filtering to Increase 20 Times of Direct Modulation Speed of White-Light LED Visible Light Communication System," Optics Express, Vol. 20, No.15, 2012, pp. 16218-16223. doi:10.1364/OE.20.016218.

[8]. R. Mesleh, H. Elgala and H. Hass, "Optical Spatial Modulation,” Journal of Optical communications and Networking, Vol. 3, No. 3, 2011, pp. 234-244. doi:10.1364/JOCN.3.000234.

[9]. T. Komine and M. Nakagawa, "Fundamental Analysis for Visible-Light Communication System using LED Lights," IEEE Transactions on Consumer Electronics, Vol. 50, No. 1, 2004, pp. 100-107. doi:10.1109/TCE.2004.1277847.

[10]. L. Zeng, D. O’Brien, H. Le-Minh, G. E. Faulkner, K. Lee, D. Jung, Y. Oh, and E. T. Won, "High Data Rate Multiple Input and Multiple Output (MIMO) Optical Wireless Communications using White LED lighting," IEEE Journal Selected Areas in Communications, Vol.27, No.9, 2009, pp.1654-1662.

[11]. H. Sugiyama, S. Haruyama, and M. Nakagawa, "Brightness control methods for illumination and visible-light communication systems," in Third International Conference on Wireless and Mobile Communications, 2007 (ICWMC'07). IEEE, 2007, pp. 78-78.

[12]. L. Ding, F. Liu, Y. He, H. Zhu and Y. Wang, "Design of Wireless Optical Access System using LED," Optics and Photonics Journal, Vol. 3 No. 2B, 2013, pp. 148-152. doi: 10.4236/opj.2013.32B036.

[13]. Y. Yang, X. Chen, L. Zhu, B. Liu and H. Chen, "Design of Indoor Wireless Communication System Using LEDs," Communications and Photonics Conference, 2-6 November 2009, Vol. 7632, pp.1-8.

[14]. Burton, C. Amiot, H. L. Minh and Z. Ghassemlooy, "Design of an integrated Optical Receiver for Mobile Visible Light Communications," PGNet, 2011.

[15]. WANG Jia-yuan, ZOU Nian-yu, WANG Dong, IRIE Kentaro, IHA Zensei, NAMIHIRA Yoshinori. Experimental study on visible light communication based on LED, The Journal of China Universities of Posts and Telecommunications, Volume 19, Supplement 2, October 2012, Pages 197-200.

[16]. Liwei Ding, Fang Liu, Yingjie He, Hongbo Zhu, Yongjin Wang, Design of Wireless Optical Access System using LED, Optics and Photonics Journal, 2013, 3, 148-152 doi:10.4236/opj.2013.32B036 Published Online June 2013 (http://www.scirp.org/journal/opj).

[17]. Angga Pradana, Nur Ahmadi, Trio Adiono, Design and Implementation of Visible Light Communication System Using Pulse Width Modulation, The 5th International Conference on Electrical Engineering and Informatics 2015 August 10-11, 2015, Bali, Indonesia.

[18]. Sung-Man Kim and Hyun-Jun Lee, Half-duplex visible light communication using an LED as both a transmitter and a receiver; INTERNATIONAL JOURNAL OF COMMUNICATION SYSTEMS Int. J. Commun. Syst. (2015) Published online in Wiley Online Library (wileyonlinelibrary.com). DOI: 10.1002/dac.2921.

[19]. Kaiyun Cui, Gang Chen, Zhengyuan Xu and Richard D. Roberts. Line-of-sight Visible Light Communication System Design and Demonstration, Communication Systems Networks and Digital Signal Processing (CSNDSP), 2010 7th International Symposium on (CSNDSP), pp. 621-625, 2010. 